\title{
Energy Efficient Clustering Routing Protocol based on LEACH for WSN
}

\author{
Shreesha Bhat \\ Department of ISE \\ NMAMIT, Nitte, VTU Belgam
}

\author{
Vasudeva Pai \\ Department of ISE \\ NMAMIT Nitte, VTU Belgam
}

\author{
Pranesh V Kallapur \\ Department of ISE \\ NMAMIT Nitte, VTU Belgam
}

\begin{abstract}
Wireless Sensor Networks are made up of restricted energy driven resources. When the installation setup of sensor nodes are over usually they are inaccessible to the user. Hence the replacement of battery source is not feasible. In order to boost the life span of the network, the key design issue is to be enhanced which is energy efficiency. With the restricted energy supply, a number of network layer protocol have been anticipated to increase the operative life span of the network. LEACH(Low Energy Adaptive Clustering Hierarchy) is a Hierarchical Clustering Protocol provides solution for such issues. EEACRA(Energy Efficient Adaptive Cluster Routing Algorithm) a variant of LEACH have been proposed to improve the deficiency of LEACH which is based on the energy decision condition.The proposed EECRL(Energy Efficient Clustering Routing for LEACH)shows several improvement for whole network energy consumption and effectively prolong the network life cycle.
\end{abstract}

\section{Keywords: EEACRA, EECRL, LEACH, Cluster head}

\section{INTRODUCTION}

The WSN(Wireless Sensor Network) embodies sensor nodes to gather information from the physical environment, process and transmit it to the destination base station. A sensor node can screen set of given natural conditions, such as sound, temperature, light or vibrations. Microcontroller, sensors, radio handset and battery are the segments of a sensor node. Multiple usefulness and simple arrangement are the advantages of sensor node[1].Modern sensor systems allow an extensive number of sensor nodes to be haphazardly sent in blocked off , unattended zones as a result of their physical dimension. However energy and system lifetime are main consideration in sensor network. In request to exploit highlights of sensor node, certain imperatives connected with them needs to be taken into account. Since the sensor nodes have tiny ,irreplaceable batteries with restricted force ability, it is important to have a system with energy effective and most extreme system lifetime. To allay such issue numerous routing conventions have been proposed. Right now, WSN routing conventions can be principally separated into two classifications, i.e., level directing conventions and various levelled directing conventions. In a moderately ideal various levelled structure, the neighbouring nodes are bunched and after that a bunch head node, which is in charge of overseeing nodes in the group and corresponding with the base station, can be picked. Such a various levelled structure can lessen the correspondence cost, as well as use the group head node with higher energy to gather the information in the bunch's scope in order to spare energy and delay the life of the system.

\section{LITERATURE SURVEY}

$\mathrm{LEACH}$ is a various levelled grouping routing convention which gives most extreme system lifetime and energy efficiency. This convention is proposed by Massachusetts Institute of Technology(MIT) electronic building and Computer Science Institute. Based on multilevel structure this is the first convention applied[2,3,4].This utilizes the method of arbitrarily pivoting the part of a group head among all the nodes in the system. The operation of LEACH is composed in rounds where each round comprises of a setup stage and a transmission stage. Amid the setup stage, the nodes sort out themselves into bunches with one node serving as the bunch head in every group. The choice to turn into a bunch head is made provincially inside of every node, and a foreordained rate of the nodes serve as nearby group heads in each round, overall. Amid the transmission stage, the self chose group heads gather information from nodes inside of their individual bunches and apply information combination before sending them straightforwardly to the base station. Toward the end of a given round, a new arrangement of nodes gets to be group sets out toward the ensuing round. Moreover, the length of time of the transmission stage is situated much bigger than that of the setup stage keeping in mind the end goal to counterbalance the overhead because of bunch arrangement. In this way, LEACH gives a decent model where confined calculations and information collection can be performed inside haphazardly self-chose bunch heads, which help decrease data over-burden and give a dependable arrangement of information to the end user. But still LEACH has a few weaknesses:

1.Additional overhead acquired while transforming groups at every set-up stage.

2..Random choice and uneven dispersion of group heads as arbitrary determination of bunch heads bring about group heads being spread in a brought together range. In this way prompting long transmission separation and wastage of energy.

3.There is no procurement of selecting another group head if there should be an occurrence of sudden disappointment of bunch head.

To defeat the disadvantages, numerous enhanced calculations have been proposed. Whereas ,these calculations principally focus on the advancement issues of the bunch heads rate and appropriation objectivity, and moreover, infrequently consider the information transmission way enhancement. In light of the inside and out investigation of LEACH, we propose a novel enhanced calculation EEACRA. 


\subsection{Network model}

In our exploration, we utilize the single order system model indistinguishable with the one of LEACH with suspicions as take after:

- All nodes are the same, static, and have enough figuring ability to backing diverse MAC conventions and information preparing.

- The radio sign has indistinguishable energy weakening in all headings, and the remote channel is symmetric.

- All nodes can speak with one another and the sink in single bounce mode.

- All nodes can be mindful they could call their own remaining energy and adjust transmission force as per correspondence separations.

- Sinks are static, and with enough power supply.

- Each node transmits information at given time opening. The information detected by adjoining nodes are correlative, so the bunch head can meld the aggregate .

\subsection{Energy model}

We utilize the first request radio model here. To transmit a kbit information to a separation $\mathrm{d}$, the radio equipment energy utilization is:

$\mathrm{E}_{\mathrm{TX}}(\mathrm{k}, \mathrm{d})=\mathrm{E}_{\mathrm{TX} \text {-elec }}(\mathrm{k})+\mathrm{E}_{\mathrm{TX} \text {-amp }}(\mathrm{k}, \mathrm{d})$

$$
\left\{\begin{array}{cc}
\text { Eelec } * k+\varepsilon f s * k * d^{\wedge} 2 & d<d o \\
\text { Eelec } * k+\text { Emp } * k * d^{\wedge} 4 & d \geq d o
\end{array}\right.
$$

where Eelec is the factor of electronics energy consumption, $\varepsilon_{\mathrm{fs}}$ and $\varepsilon_{\mathrm{mp}}$ are identical to the ones in (2), do is the reference distance between transmitter and receiver, which is given by square root of $\varepsilon_{\mathrm{fs}} / \varepsilon_{\mathrm{mp}}$.

To receive a k-bit data, the radio expends:

$E_{R X}(k)=E_{\text {elec }} \times k$

It is expected that the detected data is associated, consequently group head can simply total the information accumulated from its individuals into a solitary length-altered bundle. Bunch head totals k-bit information from $n$ individuals to consume:

$E(k, n)=n \times E_{D A} \times k$

where $\mathrm{E}_{\mathrm{DA}}$ is the factor of data aggregation .

\section{THE EXISTING EEACRA OF LEACH}

In the setup stage, every node chooses whether or not to turn into a bunch head for current round. The determination relies on upon choice made by the node by picking an arbitrary number somewhere around 0 and 1 . On the off chance that the number is not exactly the limit $\mathrm{T}(\mathrm{n})[5]$, the node turns into a group head for the present round. The limit is situated as:

$T(n)=\left\{\begin{array}{c}\frac{p}{1-p\left(r \bmod \left(\frac{1}{p}\right)\right)}, \text { if } n \in G \\ 0, \text { otherwise }\end{array}\right.$

Where $\mathrm{p}$ is the likelihood of the node being chosen as a group head node, $r$ is the quantity of rounds passed, and $G$ is the situated of nodes that have not been bunch heads in the last
$1 / \mathrm{p}$ rounds, mod signifies modulo administrator. Nodes that are group heads in round $\mathrm{r}$ should not be chosen in the following $1 / \mathrm{p}$ rounds. Accept that the starting node number $\mathrm{N}_{0}$, and the ideal bunch head rate or likelihood is Kep/ $\mathrm{N}_{0}$. As indicated by comparison, $\mathrm{K}_{\mathrm{opt}}$ is in extent to square base of the living node number $\mathrm{N}$, and backwards extent to $\mathrm{N}$. Consequently the enhanced bunch head likelihood is:

$P e p=\frac{k e p}{\sqrt{N o \sqrt{N}}}$

where $\mathrm{N}_{0}$ is the introductory node number, $\mathrm{N}$ is the living node number. $\mathrm{N}$ is equivalent to $\mathrm{N}_{0}$ if no node fails.Based on Pep, we present energy weighted variable and energy choice limit. So the enhanced group head race edge can be communicated as:

$$
\begin{gathered}
\operatorname{Tep}(n)=\left\{\begin{array}{c}
\text { pep } \times E n_{-} c u r \times \frac{\sigma}{E c h \_a v(r-1)}, \quad \beta \geq 0.5 \\
0, \quad \beta<0.5
\end{array}\right. \\
\operatorname{Tep}(n)=\left\{\begin{array}{c}
\text { kep } \times E n_{-} c u r \times \frac{\sigma}{E c h \_a v *(r-1) \sqrt{N o \sqrt{N}}}, \quad \beta \geq 0.5 \\
0, \beta<0.5
\end{array}\right.
\end{gathered}
$$

Where $\beta=\frac{E n_{-} \text {cur }}{E c h \_a v(r-1)}$

where Pep is the bunch head decision likelihood of node $n$ in current round, $\beta$ is energy weighted element, En-mutt is the lingering energy of node $n$, Ech_av(r-1) is normal leftover energy of all nodes in last round; $r$ is the present round number[6]; $\sigma$ is amendment variable. The routing calculation is in light of Selecting the ideal hand-off bunch heads by figuring introductory way cost,updating the way cost lastly keeping up the ideal way.

\section{PROPOSED EECRL FUNCTIONING}

The EEACRA uses the threshold which is same as the LEACH.In LEACH the selection is in random fashion,so the lower residual nodes could be elected as the cluster head in the next round.To avoid lower energy nodes to be selected as the cluster head the residual energy is used to find the cluster head for the next round.The threshod is changed as

$T(n)=\left\{\begin{array}{c}\frac{p}{1-p\left(r \bmod \left(\frac{1}{p}\right)\right)} * p\left(\frac{\text { Eavg }}{\text { Ecur }}\right), \text { if } n \in G \\ 0, \text { otherwise }\end{array}\right.$

Where $p\left(\frac{\text { Eavg }}{\text { Ecur }}\right)=\left\{\begin{array}{c}\frac{(\text { Ecur-Eavg })}{\text { Ecur }, \text { if Eavg }<\text { Ecur }} \\ \text { 0, otherwise }\end{array}\right.$

Here $E_{a v g}$ is the average residulal node enrgy for a round.The selection of cluster head based on the energy decision threshold is modified using logarithm function on energy weighed factor as

$\operatorname{Tep}(n)=\left\{\begin{array}{c}\log (\beta)+P e p * \sigma, \beta \geq 0.5 \\ 0, \beta<0.5\end{array}\right.$

The routing calculation utilized as a part of EEACRA is in view of expense methodology which is tedious and postures extra upkeep of directing talble. But to dodge it in EECRL we utilize energy qualities got by the bunch head .The group head finds the greatest energy values by keeping up a list. So the 
present group head sends Task Handover message to next bunch head with most extreme energy. Nodes send information at their particular times till the following change time.

\section{SIMULATION RESULTS}

In this area, we fundamentally utilize reproductions to dissect and assess the execution of the calculation. Here Matlab is

utilized as apparatus to recreate all the strategy. To confirm the enhanced calculation proposed, we will come close the outcomes with EEACRA. The fundamental recreation parameters for our model are specified in Table I. The analysis is done by utilizing the same energy source whose starting energy is $0.5 \mathrm{~J}[7,8]$. The combination coefficient is 0.5 . Each node transmits a $\mathrm{k}$ bits information parcel every round to its bunch head. A hundred sensor nodes are orchestrated arbitrarily in the field of $100 \mathrm{~m} \times 100 \mathrm{~m}$ square meters. The sink node position is changed likewise $[9,10]$.

Table 1. Transmission Parameters value

\begin{tabular}{|c|c|c|}
\hline Description & Symbol & Value \\
\hline Number of nodes & $\mathrm{N}_{\mathrm{o}}$ & 100 \\
\hline The initial node energy & $\mathrm{E}_{\text {initial }}$ & $0.5 \mathrm{~J}$ \\
\hline $\begin{array}{l}\text { Energy consumed by the } \\
\text { amplifier to transmit at a short } \\
\text { distance }\end{array}$ & $\mathrm{E}_{\mathrm{fs}}$ & $10 \mathrm{pJ} / \mathrm{bit} / \mathrm{m}^{2}$ \\
\hline $\begin{array}{l}\text { Energy consumed by the } \\
\text { amplifier to transmit at a } \\
\text { longer distance }\end{array}$ & $\mathrm{E}_{\mathrm{mp}}$ & $0.0013 \mathrm{pJ} / \mathrm{bit} / \mathrm{m}^{4}$ \\
\hline $\begin{array}{l}\text { Energy consumed in the } \\
\text { electronics circuit to transmit } \\
\text { or receive the signal }\end{array}$ & $\overline{\mathrm{E}_{\text {elec }}}$ & $50 \mathrm{pJ} / \mathrm{bit}$ \\
\hline Data packet & $\mathrm{k}$ & 4000 bits \\
\hline Control packet & $\mathrm{L}_{\text {ctrl }}$ & 100 bits \\
\hline Data aggregation energy & $\mathrm{E}_{\mathrm{DA}}$ & $5 \mathrm{pJ} / \mathrm{bit}$ \\
\hline $\begin{array}{c}\text { The cluster probability of } \\
\text { LEACH }\end{array}$ & $\mathrm{p}$ & 0.05 \\
\hline The Sensing area & $\mathrm{M} \times \mathrm{M}$ & $100 \mathrm{~m} \times 100 \mathrm{~m}$ \\
\hline
\end{tabular}

We have considered following parameters for EEACRA and EECRL variants:

$>$ Rounds v/s No of data signals received at BS

$>$ Rounds v/s Total energy dissipation

$>$ Rounds v/s Number of nodes alive

$>$ Number of nodes $\mathrm{v} / \mathrm{s}$ Network Lifetime

$>$ Network Lifetime v/s Base-Station Location

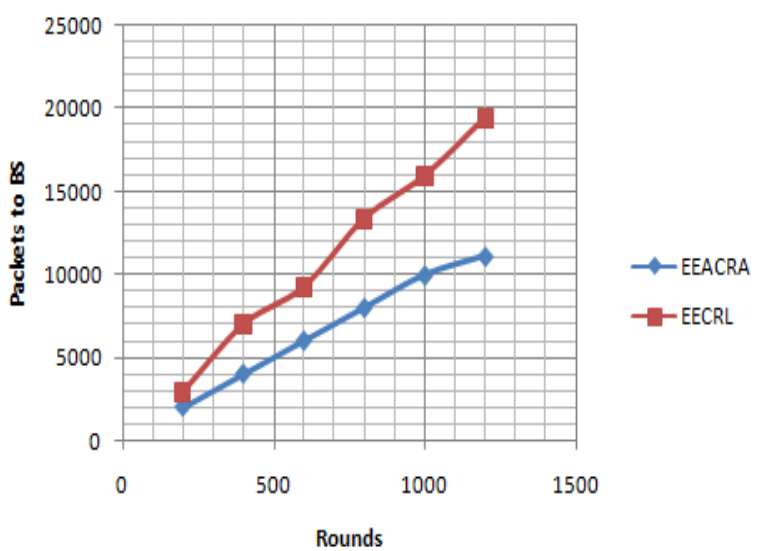

Fig.1 Rounds v/s No of data signals received at BS

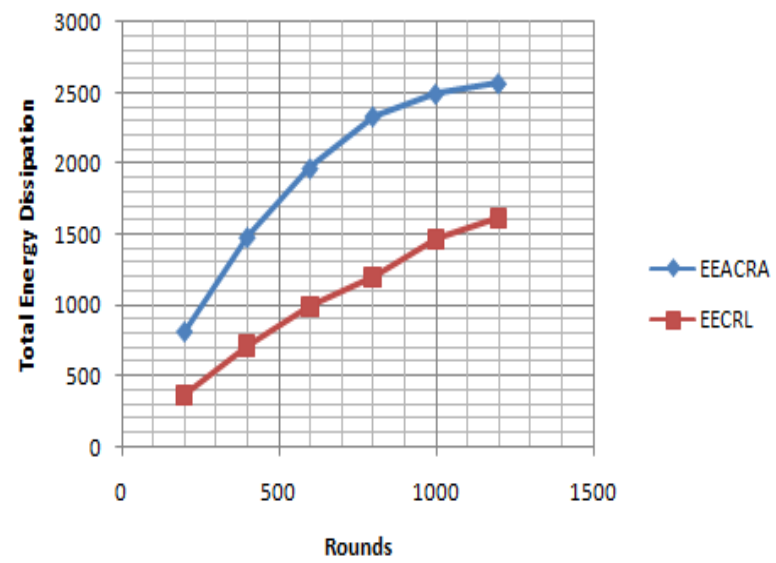

Fig.2 Rounds v/s Total energy dissipation

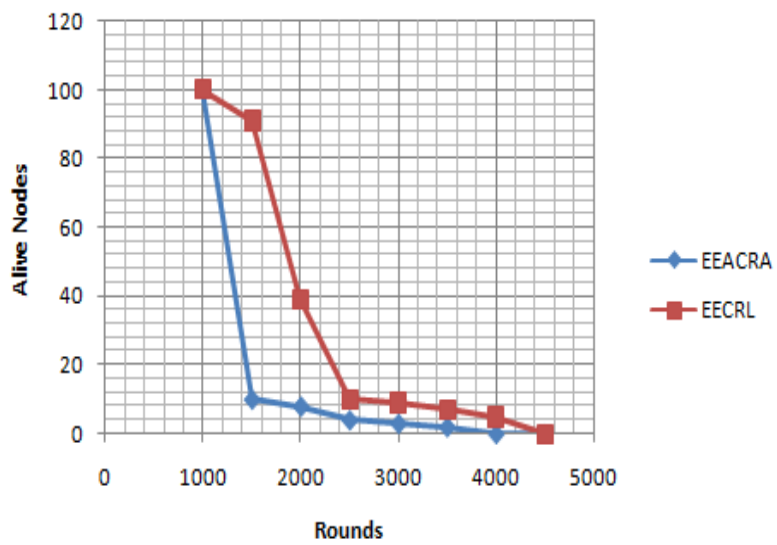

Fig 3 Rounds v/s Number of nodes alive 


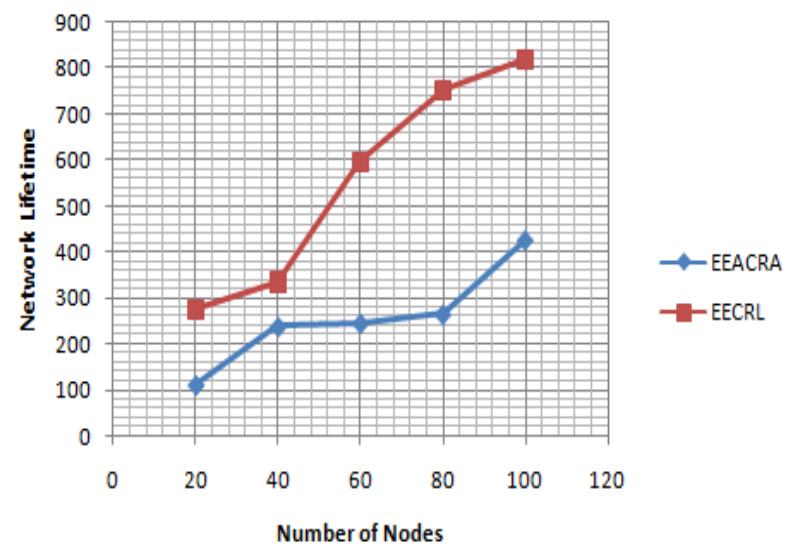

Fig. 4 Number of nodes v/s Network Lifetime

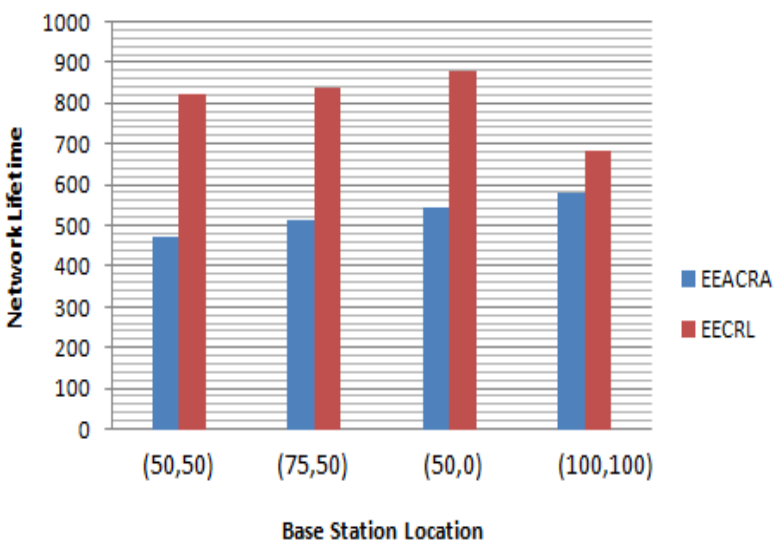

Fig. 5 Network Lifetime v/s Base-Station Location

\section{ANALYSIS OF RESULTS}

The outcome in figure1 demonstrates that packets conveyed to the base station is more in EECRL because of the enhanced edge having proficient cluster-head pivot by lingering energy of the nodes. It is seen from the figure 2 that aggregate energy dispersal is less if there should arise an occurrence of EECRL. The reason is adjusted energy choice limit by presenting energy measuring factor. Figure 3 passes on that the quantity of living nodes are less in EEACRA, since EECRL utilizes ideal group head appropriation all through the node relying upon sink location. Figure 4 briefs that system shakiness of EECRL is shorter, and when the system is near to its lifetime, the nodes come up short speedier, so EECRL can successfully adjust energy consumption. The $53.7 \%$ of nodes kick the bucket quicker in EEACRA than EECRL as a result of the routing of parcels in view of the energy got in the bunch head. According to figure 5 Network lifetime is more in base station location $(50,0)$ but in case of EEACRA it is moderately good.

\section{CONCLUSION}

In view of the exploration of LEACH, a novel convention EEACRA for WSNs was proposed. EEACRA acquires the benefits of LEACH including the grouping, bunch head pivot, information combination component, and makes enhancements by presenting of energy weighted figure group head decision threshold.But despite everything it utilizes the cocept of LEACH which does not takes care of the issue that the lower energy nodes getting to be Cluster head for next round.In expansion it utilizes the expense based way to deal with course parcels which is prolonged and postures issues in keeping up directing table. Where as the proposed calculation
EECRL utilizes leftover energy of nodes to effectively choose the group head.The adjusted energy choice edge and routing in light of most extreme energy got by group head gives great reenactment result. The reproduction results demonstrate that, contrasted and EEACRA, EECRL can further adjust the entire system energy utilization, and draw out the soundness and lifetime of the system. EECRL has more prominent energy effectiveness and ecological flexibility. The future scope is that there is still possibility of modification in the mathematical model related to selection of cluster head threshold.

\section{ACKNOWLEDGMENT}

The authors wish to acknowledge all of the teachers of NMAMIT, Nitte who guided by their knowledge and support.

\section{REFERENCES}

[1] C. Y. Chong and S. P. Kumar, "Sensor Networks: Evolution, Opportunities and Challenges", Proceedings of the IEEE, 91, No. 8, pp. 1247-1256, Aug 2003.

[2] Jianguo Shan, Lei Dong, Xiaozhong Liao, Liwei Shao, Zhigang Gao, Yang Gao,"Research On Improved Leach Protocol Of Wireless Sensor Networks", Przeglad Elektrotechniczny, 2013

[3] J.Gnanambigai,Dr.N.Rengarajan,K.Anbukkarasi,’'LEACH and Its Descendant Protocols:A Survey, International Journal of Communication and ComputerTechnologies" Volume 01 - No.3, Issue: 02 September 2012

[4] Siva d. Muruganathan, daniel c. F. Ma, rolly i. Bhasin, and abraham o. Fapojuwo, "A centralized energyefficient routing protocol for wireless sensor networks", ieee radio communications, march 2005

[5] Jia Xu,Ning Jin, Xizhong Lou,Ting Peng,Qian Zhou, Yanmin Chen, "Improvement of LEACH protocol for WSN", 9th International Conference on Fuzzy Systems and Knowledge Discovery,2012

[6] D. Mahmood, N. Javaid, S. Mahmood, S. Qureshi3, A. M. Memon, T. Zaman5,"MODLEACH: A Variant of LEACH for WSNs", Eighth International Conference on Broadband, Wireless Computing, Communication and Applications, 2013

[7] Laveena Mahajan, Narinder Shanna," Improving the Stable Period ofWSN using Dynamic Stable Leach Election Protocol" International Conference on Issues and Challenges in Intelligent Computing Techniques (ICICT), 2014

[8] Jin Ke-yin ZHANG Yao TIAN De-run, "Based on the improvement of LEACH protocol for wireless sensor network routing algorithm", Second International Conference on Intelligent System Design and Engineering Application,2012

[9] Geetha. V.a, Pranesh.V. Kallapurb, Sushma Tellajeerac, "Clustering in Wireless Sensor Networks: Performance Comparison of LEACH \& LEACH-C Protocols Using NS2", Procedia Technology 4,2012

[10] Jian-guang Jia, Zun-wen He, Jing-ming Kuang, and CunXiang Chen, "An Energy efficient Adaptive Clustering Routing Algorithm for Wireless Sensor Networks", Cross Strait Quad-Regional Radio Science and Wireless Technology Conference,2011 